\title{
Amplified Spontaneous Emission and Waveguiding Properties of the Colored Merocyanine Form of $\left(1^{\prime}, 3^{\prime}\right.$-Dihydro-1', $3^{\prime}, 3^{\prime}$-trimethyl-6- nitrospiro[2H-1-benzopyran-2,2'-(2H)-indole] Molecules
}

\author{
Luana Persano,${ }^{\dagger}$ Elisa Mele,${ }^{\dagger}$ Athanassia Athanassiou, ${ }^{\ddagger}$ Roberto Cingolani,${ }^{\dagger}$ and \\ Dario Pisignano*,† \\ NNL, National Nanotechnology Laboratory of INFM-CNR, clo Palazzine Garrisi, Università di Lecce, via \\ Arnesano, I-73100 Lecce, Italy, and Institute of Electronic Structure and Laser (IESL), Foundation for \\ Research and Technology-Hellas (FORTH), 71110 Heraklion, Crete, Greece
}

Received February 21, 2006. Revised Manuscript Received May 18, 2006

\begin{abstract}
We report a complete study of the properties of amplified spontaneous emission (ASE) of the merocyanine form of the photochromic system, 1', $3^{\prime}$-dihydro-1', $3^{\prime}, 3^{\prime}$-trimethyl-6-nitrospiro[2H-1benzopyran-2,2'-(2H)-indole], under nanosecond excitation conditions. ASE line narrowing is clearly observed for excitation densities larger than $400 \mu \mathrm{J} \mathrm{cm}^{-2}$ with a threshold pumping length in the range 0.5-1.2 $\mathrm{mm}$. Remarkable waveguiding properties were observed, with losses throughout the organic slab of about $5.7 \mathrm{~cm}^{-1}$. The observation of ASE is discussed in terms of a possible $S_{1}$-dominated photoconversion and excitation/de-excitation dynamics of the photochromic system. These results are important in view of the application of merocyanine-based films as active layers for potentially gateable laser devices.
\end{abstract}

\section{Introduction}

Highly fluorescent conjugated materials, such as polymers and low-molar-mass molecules, have been increasingly investigated in the past decade, because of their appealing features for the realization of active optical devices and especially solid-state plastic lasers and optical amplifiers. ${ }^{1}$ Conjugated systems exhibit high photoluminescence (PL) efficiency, tunability of the emission wavelength from the near-ultraviolet (UV) to the near-infrared, and high processability by cheap and simple deposition and patterning techniques. The observation of optical gain by stimulated emission in organic compounds, ${ }^{2}$ especially in thin-film form, ${ }^{3}$ has further raised their interest, in view of the realization of organic semiconductor lasers, eventually electrically driven. A number of conjugated compounds have been demonstrated to show optical gain, such as fluorenebased polymers, ${ }^{4}$ poly(para-phenylene) ( $m$-LPPP) ${ }^{5,6}$ and poly (para-phenylene vinylene $)^{7,8}$ derivatives, various small-

* Corresponding author. E-mail: dario.pisignano@unile.it.

$\dagger$ National Nanotechnology Laboratory of INFM-CNR.

\# Foundation for Research and Technology-Hellas.

(1) (a) Tessler, N. Adv. Mater. 1999, 11, 364. (b) Kranzelbinder, G.; Leising, G. Rep. Prog. Phys. 2000, 63, 729. (c) McGehee, M. D.; Heeger, A. J. Adv. Mater. 2000, 12, 1655.

(2) Moses, D. Appl. Phys. Lett. 1992, 60, 3215.

(3) Tessler, N.; Denton, G. J.; Friend, R. H. Nature 1996, 382, 695.

(4) Xia, R.; Heliotis, G.; Bradley, D. D. C. Appl. Phys. Lett. 2003, 82, 3599.

(5) Graupner, W.; Leising, G.; Lanzani, G.; Nisoli, M.; De Silvestri, S.; Scherf, U. Phys. Rev. Lett. 1996, 76, 847.

(6) Stagira, S.; Nisoli, M.; Cerullo, G.; Zavelani-Rossi, M.; De Silvestri, S.; Lanzani, G.; Graupner, W.; Leising, G. Chem. Phys. Lett. 1998, $289,205$.

(7) Frolov, S. V.; Gellerman, W.; Ozaki, M.; Yoshino, K.; Vardeny, Z. V. Phys. Rev. Lett. 1997, 78, 729.

(8) Denton, G. J.; Tessler, N.; Stevens, M. A.; Friend, R. H. Adv. Mater. 1997, 9, 547 . molecular-weight organic blends, ${ }^{9}$ and substituted oligothiophenes..$^{10}$ The feedback mechanism for optically pumped lasing action can be accomplished also by different geometries, such as microrings, ${ }_{1}^{11}$ microdroplets, ${ }^{12}$ planar and circular distributed Bragg reflectors, ${ }^{13}$ vertical microcavities, ${ }^{3}$ and distributed feedback resonators. ${ }^{14}$

For a conjugated compound to be used as an active medium of a laser, many conditions have to be fulfilled. Hence, the realization of these devices requires a lot of efforts both from the chemistry and the nanofabrication points of view. Basic prerequisites are a high luminescence efficiency and a large separation between the spectral regions of gain and absorption (native and photoinduced). Large Stokes' shift, that is, low self-absorption, strongly favors the lasing action. ${ }^{15}$ The lasing condition for conjugated compounds is often fulfilled by a four-level scheme based on the vibronic

(9) (a) Kozlov, V. G.; Buloviæ, V.; Burrows, P. E.; Forrest, S. R. Nature 1997, 389, 362. (b) Bergreen, M.; Dodabalapur, A.; Slusher, R. E.; Bao, Z. Nature 1997, 389, 466.

(10) Pisignano, D.; Anni, M.; Gigli, G.; Cingolani, R.; Zavelani-Rossi, M.; Lanzani, G.; Barbarella, G.; Favaretto, L. Appl. Phys. Lett. 2002, 81, 3534.

(11) Frolov, S. V.; Fujii, A.; Chinn, D.; Vardeny, Z. V.; Yoshino, K.; Gregory, R. V. Appl. Phys. Lett. 1998, 72, 2811.

(12) Berggren, M.; Dodabalapur, A.; Bao, Z.; Slusher, R. E. Adv. Mater. 1997, 9, 968.

(13) (a) Berggren, M.; Dodabalapur, A.; Slusher, R. E. Appl. Phys. Lett. 1997, 71, 2230. (b) Moll, N.; Mahrt, R. F.; Bauer, C.; Giessen, H.; Schnabel, B.; Kley, E. B.; Scherf, U. Appl. Phys. Lett. 2002, 80, 734.

(14) (a) McGehee, M. D.; Diaz-García, M. A.; Hide, F.; Gupta, R.; Miller, E. K.; Moses, D.; Heeger, A. J. Appl. Phys. Lett. 1998, 72, 1536. (b) Turnbull, G. A.; Andrews, P.; Jory, M. J.; Barnes, W. L.; Samuel, I. D. W. Phys. Rev. B 2001, 64, 125122-1. (c) Riechel, S.; Lemmer, U.; Feldmann, J.; Berleb, S.; Mückl, A. G.; Brütting, W.; Gombert, A.; Wittwer, V. Opt. Lett. 2001, 26, 593. (d) Pisignano, D.; Persano, L.; Visconti, P.; Cingolani, R.; Gigli, G.; Barbarella, G.; Favaretto, L. Appl. Phys. Lett. 2003, 83, 2545.

(15) Hide, F.; Diaz-García, M. A.; Schwartz, B. J.; Andersson, N. R.; Heeger, A. J. Science 1996, 273, 1833. 
progressions of the electronic ground state, $S_{0}$, and the firstexcited electronic state, $S_{1}$. The population inversion is indeed accomplished by the photoexcitation from the ground vibrational level of $S_{0}$ to a vibrationally hot sublevel of $S_{1}$, followed by the subsequent rapid decay to the ground vibrational level of $S_{1}$, by Internal Conversion. ${ }^{1}$ For cavities with effective feedback, the lasing threshold in conjugated polymers and low-molar-mass molecule systems is limited by the radiation losses along the cavity, including both selfabsorption and scattering losses. Because in organic optoelectronic devices, and especially optical amplifiers, a crucial role is played by the waveguiding capability of the active films, the determination of the waveguide performances of active organic slabs is of particular interest.

A strategic class of conjugated molecules that so far have not been investigated as possible media for stimulated emission and optical gain are given by the photoinduced color forms of photochromic compounds. Photochromism, ${ }^{16}$ namely, the reversible photoinduced transformation of molecular structures, is attracting increasing interest because of its possible application to optical devices and memories, ${ }^{17}$ switchable molecular recognition, ${ }^{18}$ gateable volume changes for photoactuation, ${ }^{19}$ and interfacial properties for analytical chemistry and microfluidics. ${ }^{20}$ In particular, spiropyran molecules ${ }^{21}$ such as the indolinospiropyran $\left(1^{\prime}, 3^{\prime}\right.$-dihydro$1^{\prime}, 3^{\prime}, 3^{\prime}$-trimethyl-6-nitrospiro[2H-1-benzopyran-2,2'-( $\left.2 H\right)$-indole]) (6-nitro-BIPS) are initially nonpolar and transparent at visible wavelengths and absorb in the $\mathrm{UV}(\lambda \leq 400 \mathrm{~nm})$, whereas they are reversibly converted to the visible-absorbing $(\lambda \leq 680 \mathrm{~nm})$ isomeric form, trans-merocyanine (trans-MC), upon UV irradiation through the photochemical cleavage of the $\mathrm{C}-\mathrm{O}$ bond in the spiropyran ring (Figure 1). The MC form exhibits indeed a $\pi$-electron delocalization. Furthermore, it was found by a number of techniques that a highenergy cis-MC form acts as a transient intermediate in the transition kinetics, leading to the rearrangement to the trans

(16) Tamai, N.; Myiasaka, H. Chem. Rev. 2000, 100, 1875.

(17) (a) Tsivgoulis, G. M.; Lehn J. M. Angew. Chem., Int. Ed. 1995, 34, 1119. (b) Tamaoki, N.; Van Keuren, E.; Matsuda, H.; Hasegawa, K.; Yamaoka, T. Appl. Phys. Lett. 1996, 69, 1188. (c) Willner, I. Acc. Chem. Res. 1997, 30, 347. (d) Emmelius, M.; Pawlowski, G.; Vollmann, H. W. Angew. Chem., Int. Ed. 1998, 28, 1445. (e) Hobley, J.; Fukumura, H.; Goto, M. Appl. Phys. A 1999, 69, 945. (f) Cinelli, R. A. G.; Pellegrini, V.; Ferrari, A.; Faraci, P.; Nifosi, R.; Tyagi, M.; Giacca, M.; Beltram, F. Appl. Phys. Lett. 2001, 79, 3353. (g) Raymo, F. N.; Giordani, S. Proc. Natl. Acad. Sci. U.S.A. 2002, 99, 4941. (h) Tyson, D. S.; Bignozzi, C. A.; Castellano F. N. J. Am. Chem. Soc. 2002, 124, 4562. (i) Tsujioka, T.; Kondo, H. Appl. Phys. Lett. 2003, $83,937$.

(18) (a) Inouye, M.; Ueno, M.; Kitao, T.; Tsuchiya, K. J. Am. Chem. Soc. 1990, 112, 8977. (b) Inouye, M.; Kim, K.; Kitao, T. J. Am. Chem. Soc. 1992, 113, 778. (c) Willner, I.; Blonder, R.; Dagan, A. J. Am. Chem. Soc. 1994, 116, 3121.

(19) (a) Ikeda, T.; Nakano, M.; Yu, Y.; Tsutsumi, O.; Kanazawa, A. Adv. Mater. 2003, 15, 201. (b) Yu, Y.; Nakano, M.; Ikeda, T. Nature 2003 425, 145. (c) Athanassiou, A.; Kalyva, M.; Lakiotaki, K.; Georgiou, S.; Fotakis, C. Adv. Mater. 2005, 17, 988.

(20) (a) Freemantle, M. Chem. Eng. News 1999, 77, 27. (b) Ichimura, K.; Oh, S.; Nakagawa, M. Science 2000, 288, 1624. (c) Rosario, R.; Gust, D.; Hayes, M.; Jahnke, F.; Springer, J.; Garcia, A. A. Langmuir 2002 18, 8062. (d) Rosario, R.; Gust, D.; Garcia, A. A.; Hayes, M.; Taraci, J. L.; Clement, T.; Dailey, J. W.; Picraux, S. T. J. Phys. Chem. B 2004, 108, 12640. (e) Bunker, B. C.; Kim, B. I.; Houston, J. E.; Rosario, R.; Garcia, A. A.; Hayes, M.; Gust, D.; Picraux, S. T. Nano Lett. 2003, 3, 1724.

(21) Berkovic, G.; Krongauz, V.; Weiss V. Chem. Rev. 2000, 100, 1741. (a)

(b)

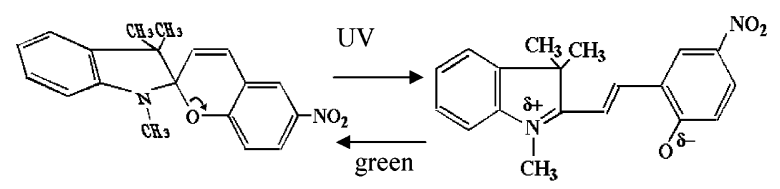

(c)

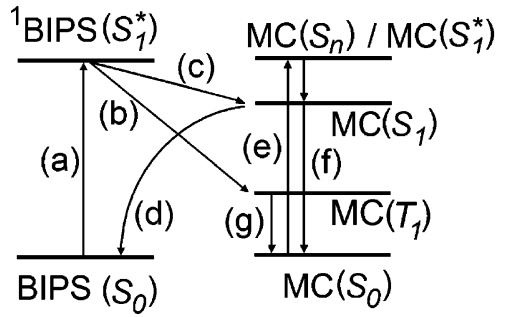

Figure 1. Reversible transformations between the closed (a) and the open (b) forms of the photochromic molecule (6-nitro-BIPS and MC, respectively), by irradiation with UV and visible light. (c) General scheme for the excitation/de-excitation dynamics of 6-nitro-BIPS upon optical pumping. The different letters in part $\mathrm{c}$ indicate the following processes: (a) optical excitation from the ground $\left(S_{0}\right)$ to the vibrationally hot $S_{1}$ BIPS state $\left(S_{1}{ }^{*}\right)$; (b and c) decay to the triplet and singlet ground $S_{1}$ BIPS states, respectively; (d) back decay to the BIPS electronic ground state; (e) excitation to vibrationally hot $S_{1}$ or $S_{n}$ MC states; (f) singlet decay to the ground MC state; and (g) triplet-singlet decay to the ground MC state. For clarity reasons only the transitions of direct interest for ASE are shown. Intermediate transitions (triplet and singlet BIPS $\rightarrow$ MC photoconversion pathways through cis-MC intermediate states, ground-state cis-MC $\rightarrow$ BIPS back-conversion, and singlet cis-MC decay to the ground electronic state) are omitted.

form in short time $(<100 \mathrm{ps}) .^{22}$ A general scheme of the BIPS $\rightleftarrows$ MC photoconversion and excitation/de-excitation is depicted in Figure 1c.

Notwithstanding the wide range of the application fields of photochromic molecules, the mechanism of the BIPSMC photochemical conversion has not been fully assessed yet. In particular, the electronic character (singlet or triplet) of the conversion is not yet completely understood. Many studies enlightened the importance of substituents in affecting the conversion dynamics. A recent comprehensive theoretical study by Sheng et al. ${ }^{23}$ found that, upon irradiation with UV light, the coloration process (given by the cleavage of the $\mathrm{C}-\mathrm{O}$ bond of the spiro moiety and the cis - trans transformation) proceeds through the excited singlet states for spiropyrans having the indoline functionalized by electronwithdrawing groups and the chromene functionalized by electron-donating groups ("acceptor-donor", such as $\mathrm{H}-\mathrm{CH}_{3}$ ), whereas the conversion is more favored via a triplet manifold for "donor-acceptor" $\left(\mathrm{H}-\mathrm{NO}_{2}, \mathrm{CH}_{3}-\mathrm{NO}_{2}\right)$ substituted compounds. This is in agreement with previous studies about ring openings involving a $\mathrm{NO}_{2}$-functionalized chromene. ${ }^{24}$

(22) (a) Görner, H.; Chibisov, A. K. J. Chem. Soc., Faraday Trans. 1998 94, 2557. (b) Chibisov, A. K.; Görner, H. Chem. Phys. 1998, 237, 425. (c) Wojtyk, J. T.; Kazmaier, P. M.; Buncle, E. Chem. Mater. 2001, 13, 2547. (d) Görner, H. Chem. Phys. Lett. 1998, 282, 381. (e) Görner, H. Chem. Phys. 1997, 222, 315. (f) Bohne, C.; Fan, M. G. Li, Z. H.; Laing, Y. C.; Lusztyk, J.; Scaiano, J. C. J. Photochem. Photobiol., A 1992, 66, 79. (g) Malatesta, A.; Neri, C.; Wis, M. L.; Montanari, L.; Millini, R. J. Am. Chem. Soc. 1997, 119, 3451. (h) Chibisov, A. K.; Görner, H. J. Phys. Chem. A 1997, 101, 4305. (i) Levitus, M.; Talhavini, M.; Atvars, T. D. Z.; Aramendia, P. F. J. Phys. Chem. B 1997, 101, 7680.

(23) Sheng, Y.; Leszczynski, J.; Garcia, A. A.; Rosario, R.; Gust, D.; Springer, J. J. Phys. Chem. B 2004, 108, 16233. 
Instead, the MC-BIPS conversion (decoloration process) was found to rely only on one singlet-driven mechanism, regardless of the specific substituent employed. ${ }^{23}$

6-Nitro-BIPS was also found to exhibit a good thermal stability and a large quantum yield of photocoloration. ${ }^{16}$ Indeed, the nitro group at the 6 position of the benzopyran moiety strongly favors the conversion to the MC form. For instance, the photocoloration efficiency is in the range 0.60.8 for molecules in solvents of low polarity at room temperature, decreasing to less than 0.2 in polar solvents, such as acetonitrile and ethanol. ${ }^{2 \mathrm{~h}} \mathrm{~A}$ remarkable effect of reduction of the photocoloration yield was also observed upon decreasing the temperature and upon increasing the medium viscosity. ${ }^{22 c}$ Embedding the photochromic molecules within possibly highly viscous polymeric matrixes for the realization of solid-state light emitters can, therefore, affect significantly the photoconversion properties. In addition, after UV irradiation the BIPS form can be recovered by green light exposure or even thermally, although the thermal decoloration within polymeric matrixes (time scale of hundreds of minutes at room temperature $)^{22 \mathrm{i}}$ can be neglected for the time scales of typical optical experiments. Concerning the fluorescence efficiency of MC, once it is formed, values of the order of $1 \%$ in solution at room temperature, increasing up to $80 \%$ at $77 \mathrm{~K}$, have been reported. ${ }^{22 \mathrm{c}}$

The importance of achieving optical gain by a particular state of photoswitchable molecules stands in the possible realization of, eventually, gateable lasing devices. We recently reported the accomplishment of optical gain by the open form of the MC resulting from the UV irradiation of 6-nitro-BIPS, embedded in a polymeric matrix at the solid state. ${ }^{25}$ In this paper, we present a complete study of the properties of amplified spontaneous emission (ASE) and waveguiding in MC-based films under pulsed optical excitation. We measured a threshold excitation density for optical gain-induced line narrowing of about $400 \mu \mathrm{J} \mathrm{cm} \mathrm{c}^{-2}$ and a threshold length in the range $0.5-1.2 \mathrm{~mm}$ and remarkable waveguiding properties (losses of about $5.7 \mathrm{~cm}^{-1}$ ). These results strongly support the use of the BIPS-MC photochromic system for realizing solid-state organic lasers.

\section{Experimental Details}

6-Nitro-BIPS/poly(ethyl methacrylate)-co-poly(methyl acrylate) (PEMMA) films were cast by spin coating from toluene solutions with a relative concentration of $10 \%(\mathrm{w} / \mathrm{w})$ on Corning quartz substrates $(n=1.46)$. With a spin-coating speed of $2000 \mathrm{rpm}$ we obtained neat films of thickness comprised between 690 and 750 $\mathrm{nm}$, measured by a profilometer. The third harmonic $(\lambda=355 \mathrm{~nm})$ of a 3 ns $Q$-switched Nd:YAG laser (Spectra-Physics, repetition rate of $10 \mathrm{~Hz}$ ) was employed as the excitation source. The pump beam was focused on the sample by a system of lenses, including a cylindrical lens providing a rectangular excitation stripe of width $500 \mu \mathrm{m}$. The sample emission at room temperature was collected from the sample edge by means of a $6-\mathrm{cm}$ diameter, $10-\mathrm{cm}$ focal length lens, then dispersed by a monochromator, and detected by a Si charge coupled device. A constant stripe length of $10 \mathrm{~mm}$

(24) Lenoble, C.; Becker, R. S. J. Phys. Chem. 1986, 90, 62.

(25) Pisignano, D.; Mele, E.; Persano, L.; Athanassiou, A.; Fotakis, C.; Cingolani, R. J. Phys. Chem. B 2006 110, 4506.

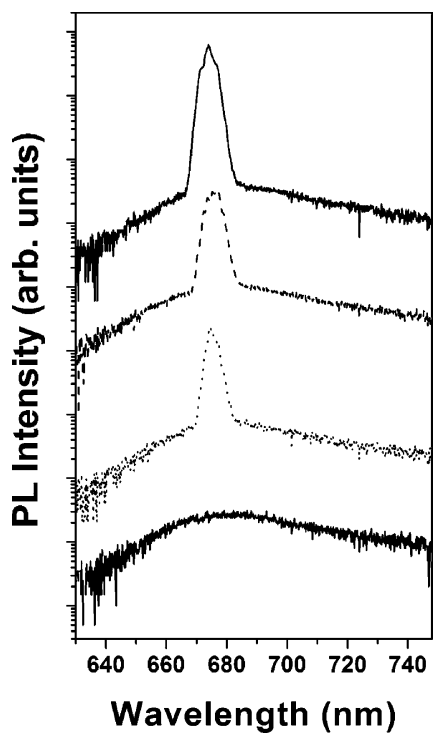

Figure 2. Emission spectra from the photochromic SP-MC/PEMMA films under different excitation fluences (from bottom to top: 0.3, 2.5, 3.7, and $6.1 \mathrm{~mJ} \mathrm{~cm}^{-2}$ ). Spectra are shifted vertically on a log scale for clarity reasons.

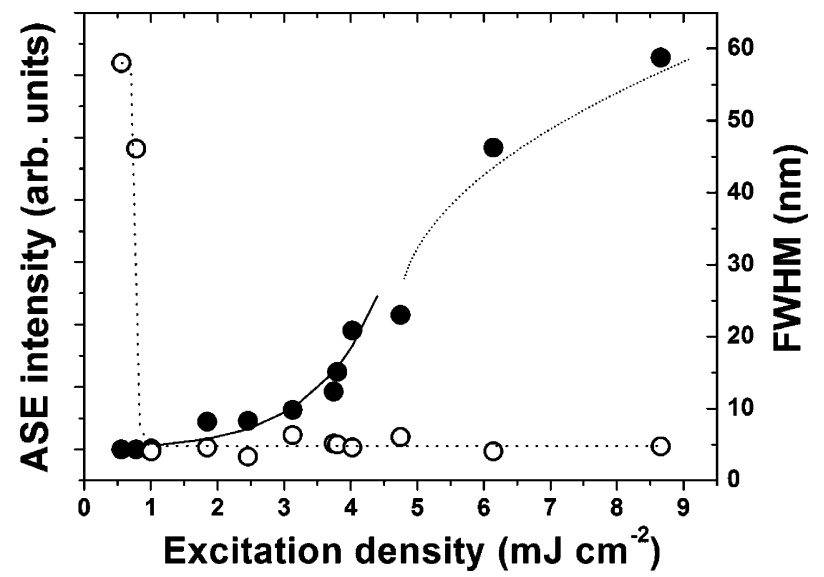

Figure 3. Dependence of the ASE integrated emitted intensity (solid circles, left scale) and spectral width (fwhm, open circles, right scale) on the pump energy density. The continuous line is the best fit to the data up to $4.0 \mathrm{~mJ}$ $\mathrm{cm}^{-2}$ by an exponential-like ASE model. The dotted line is the best fit of the data between $4.7 \mathrm{~mJ} \mathrm{~cm}^{-2}$ and $8.6 \mathrm{~mJ} \mathrm{~cm}^{-2}$ to the square root behavior predicted by the bimolecular annihilation model. The dashed curve interpolating the fwhm data is a guide for the eye only.

was employed for the measurements varying the excitation fluence. All the measurements presented in this paper were carried out under vacuum $\left(10^{-6} \mathrm{mbar}\right)$ to inhibit photo-oxidation of the active material.

\section{Results and Discussion}

In Figure 2 we show the PL spectra of 6-nitro-BIPS doped in PEMMA under pulsed optical pumping at various excitation densities $(E)$. For low values of the excitation fluence ( $E \leq 400 \mu \mathrm{J} \mathrm{cm}^{-2}$ ), we could observe only a featureless and broad (full width half-maximum, fwhm $\cong 60 \mathrm{~nm}$ ) PL spectrum, such as the bottom one in Figure 2. Instead, for larger values of $E$, a narrow peak arises from the PL spectra around $675 \mathrm{~nm}$, resulting in a significant line narrowing down to $\mathrm{fwhm}=3.3 \mathrm{~nm}$ (Figure 3).

The spectral position of the rising peak is consistent with the emission from the MC open form of the photochromic molecular system. Possible excitation-de-excitation schemes 
are through vibrationally hot states within $S_{1}$ or higher excited states $\left(S_{n}\right)$ or may involve different MC isomer states (cistrans or trans-trans), as proposed for 6,8-dinitro-BIPS in acetonitrile solutions, ${ }^{26}$ and ultimately the decay to the $S_{0}$ state of the open form. In particular, the line-narrowing can be attributed to ASE assisted by waveguiding along the organic slab. The light confinement in the direction perpendicular to the spin-cast film is indeed provided by the different refractive indexes of the substrate $\left(n_{\text {sub }}\right)$, the active material $\left(n_{\mathrm{MC}}\right)$, and vacuum $\left(n_{\mathrm{ext}}\right)$. In fact, the investigated thicknesses of the active molecular layer are well above the cutoff threshold, $t_{\text {cutoff }}$, which for a transversal electric fundamental mode is given by ${ }^{27}$

$$
t_{\text {cutoff }}=\frac{\lambda}{2 \pi \sqrt{n_{\mathrm{MC}}^{2}-n_{\text {sub }}^{2}}} \arctan \sqrt{\frac{n_{\text {sib }}^{2}-n_{\mathrm{ext}}^{2}}{{n_{\mathrm{MC}}}^{2}-n_{\mathrm{sub}}^{2}}}
$$

where the guided wavelength, $\lambda$, lies in the PL spectral region. In our experiments, we also observed a remarkable dependence of the wavelength of the ASE peak on the film thickness, namely, a red shift was found for thicker samples, which is a signature of ASE gain narrowing in conjugated organic materials. ${ }^{15}$ For instance, ASE peaked around 675 and $710 \mathrm{~nm}$ for films of thicknesses 690 and $730 \mathrm{~nm}$, respectively.

A $S_{1}$-dominated energy excitation scheme would be particularly suitable for optoelectronic applications. The ring closure kinetics of 6,8-dinitro-BIPS and its carboxyethyl derivative in acetonitrile, studied by pump-probe spectroscopy and time-resolved PL, could be explained in terms of a purely singlet state manifold. ${ }^{26}$ As a matter of fact, in our photopumped system both the forms, BIPS and MC, are present at the same time. In particular, the radiative transition originating ASE in the MC spectral region could be related to the singlet decay to the MC ground state, after the excitation to vibrationally hot $S_{1}$ or $S_{n}$ MC states, namely, the transition labeled as (e) in Figure 1c. Previously reported data collected in solution indicated a characteristic time as fast as $500 \mathrm{fs}$ for forming the excited $S_{1} \mathrm{MC}$ state from higher-energy states after photoexcitation of the ground MC state and that such an $S_{1}$ state exhibits short and long decay times (in the range of tens and hundreds of picoseconds, respectively) because of the transitions labeled as (e) in Figure $1 \mathrm{c}$ and of the back-conversion pathway, respectively. ${ }^{26}$ These values are of the same typical order of magnitude as the stimulated emission dynamics of other organic amplifiers working at the solid state, as measured by time-resolved femtosecond pump and probe experiments on $m$-LPPP (8 ps), ${ }^{5}$ poly[2-butyl-5-(2'-ethylhexyl)-1,4-phenylene vinylene] $(60 \mathrm{ps}),{ }^{28}$ and 2,5-dioctyloxy poly(para-phenylene vinylene) (240 ps). ${ }^{7}$ In MC, the $S_{1}$ excited state is very likely responsible for the population inversion at the origin of the four levels lasing scheme.

(26) Hobley, J.; Pfeifer-Fukumura, E.; Bletz, M.; Asahi, T.; Masuhara, H.; Fukumura, H. J. Phys. Chem. A 2002, 106, 2265.

(27) Marcuse, M. Theory of Dielectric Waveguides; Academic: New York, 1974.

(28) Schwartz, B. J.; Hide, F.; Andersson, M. R.; Heeger, A. J. Chem. Phys. Lett. 1997, 265, 3427.

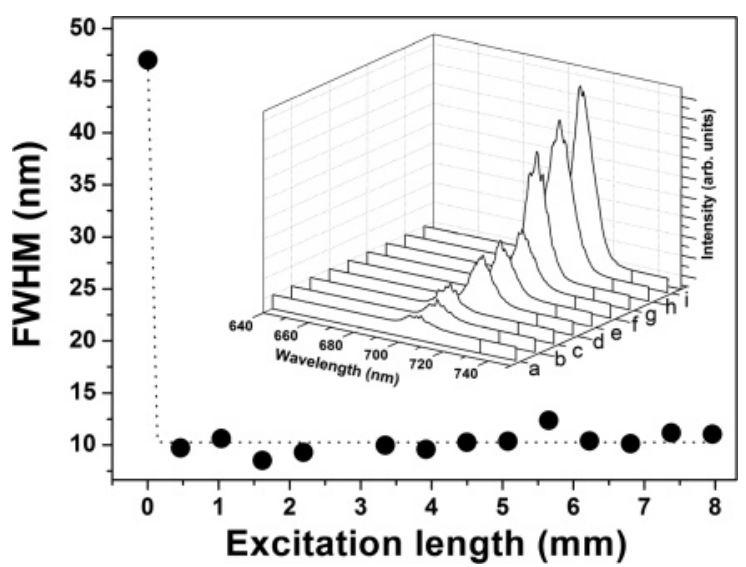

Figure 4. fwhm vs excitation length. Inset: Emission spectra by different excitation lengths: 0.47 (a), 1.04 (b), 1.62 (c), 3.35 (d), 3.92 (e), 4.50 (f), $5.08(\mathrm{~g}), 5.65(\mathrm{~h})$, and $6.23 \mathrm{~mm}(\mathrm{i})$.

The emission intensity due to the narrow band shows an abrupt (approximately exponential) increase up to excitation densities of about $4.0 \mathrm{~mJ} \mathrm{~cm}{ }^{-2}$, without the occurrence of a distinct threshold (Figure 3). For larger excitation intensities $\left(E>4.5 \mathrm{~mJ} \mathrm{~cm}^{-2}\right)$, an exponential-like behavior is no longer able to fit the experimental data, and the ASE output exhibits saturation. Such a sublinear evolution of the integrated intensity indicates the presence of nonradiative decay processes, increasing with increasing the pump density. In previous reports, the resulting gain saturation has been mainly attributed to nonlinear effects, like bimolecular exciton annihilation, induced by the high density of excitons formed under very intense photon fluxes. ${ }^{29}$ In the case of MC/PEMMA films, such a behavior, characterized by the square root dependence of the high power emission on the excitation density, may be compatible with the detected experimental trend, which seems to follow a nonlinear, asymptotic functional law, consistent with the bimolecular annihilation model (dotted curve fitting the intensity data in Figure 4 for $E>4.5$ $\left.\mathrm{mJ} \mathrm{cm}{ }^{-2}\right) .{ }^{8,29} \mathrm{We}$ found that very high excitation densities can also produce sample ablation, without a further increase of the ASE output.

To assess in depth the gain performances of the MC/PEMMA system, we also carried out measurements by the variable stripe length method, which provides the optical gain spectrum, $g(\lambda)$, of the active medium through the relation between the emitted intensity, $I$, and the excitation length, $l: 30$

$$
I(\lambda)=I_{0}(\lambda)\{\exp [g(\lambda) l]-1\}
$$

The PL spectra collected with different excitation stripes are reported in the inset of Figure 4. It can be clearly observed that the line narrowing is enhanced by longer pump lengths (Figure 4). The fwhm values suddenly drop from 47 to less than $10 \mathrm{~nm}$ upon increasing the excitation length around 0.5 $\mathrm{mm}$, and then it stabilizes. Figure 5 shows the output intensity at the peak of the emission spectrum $(710 \mathrm{~nm})$ for a 750 nm-thick MC/PEMMA film, as a function of the pump stripe length, at an excitation fluence of $0.5 \mathrm{~mJ} \mathrm{~cm}^{-2}$. The ASE

(29) Maniloff, E. S.; Klimov, V. I.; McBranch, D. W. Phys. Rev. B 1997, $56,1876$.

(30) Shaklee, K. L.; Nahory, R. E.; Leheny, R. F. J. Lumin. 1973, 7, 284. 


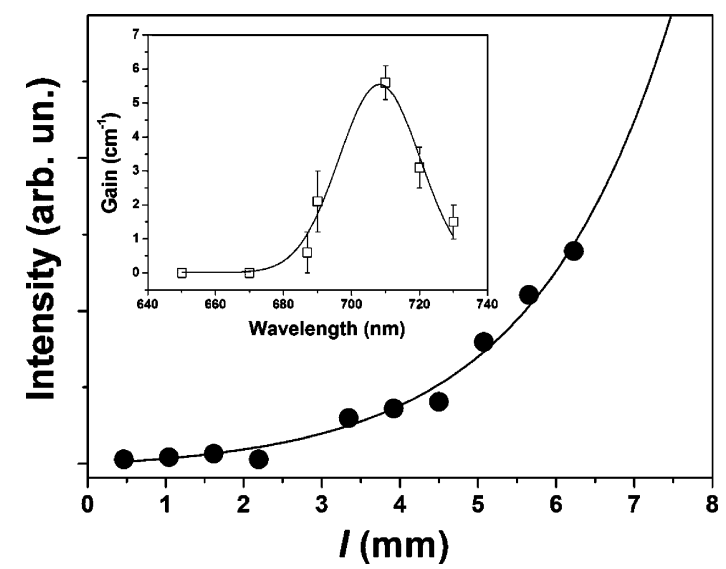

Figure 5. Dependence of the emission intensity at a wavelength of 710 $\mathrm{nm}$ on the excitation length, for a film of thickness $750 \mathrm{~nm}$. The solid line is a fit to experimental data according to eq 2. Inset: Optical gain spectrum for an excitation density of $0.5 \mathrm{~mJ} \mathrm{~cm}^{-2}$. The continuous line is a guide for the eye.

intensity increases by about 50 times upon increasing the excitation length from 0.4 to $6.2 \mathrm{~mm}$. In such an investigated range, the experimental data are well-described by eq 2 . The corresponding optical gain spectrum, $g(\lambda)$, is displayed in the inset of Figure 5, showing a well-defined peak, centered at about $710 \mathrm{~nm}$ with a spectral width of about $28 \mathrm{~nm}$ and a maximum around $5.6 \mathrm{~cm}^{-1}$. By the value of the maximum optical gain, one can extract the threshold length, $I_{\mathrm{th}}$, needed to clearly distinguish ASE, via the relation $\exp \left[g(\lambda) l_{\mathrm{th}}\right]-1$ $=1$, that is, $I_{\text {th }}=\ln 2 / g(\lambda)$. We obtain for our photochromic system $l_{\text {th }}=1.2 \pm 0.1 \mathrm{~mm}$, which is in good agreement with the experimental finding about line narrowing (Figure 4). Moreover, by the maximum optical gain one can estimate the stimulated emission cross section:

$$
\sigma_{\mathrm{g}}=\frac{\mathrm{g}}{N}
$$

where $N$ is the excitation density. We obtain $\sigma_{\mathrm{g}}=2.4 \times$ $10^{-19} \mathrm{~cm}^{2}$ (with $N=2.3 \times 10^{19} \mathrm{~cm}^{-3}$ ), which is significantly larger than the value obtained by fitting the ASE output behavior, although still 1 order of magnitude lower than the typical values of some prototype conjugated oligomers and polymers, such as thienyl-S,S-dioxide compounds, ${ }^{31}$ poly(arylene vinylene)s, ${ }^{29}$ and poly(phenylene vinylene)s. ${ }^{8}$ However, we point out that this calculation assumes unity quantum efficiency for the emitting species (namely, the stimulated emission cross section is determined by assuming $N$ equal to the number of absorbed photons per unit volume), so it underestimates the actual value of the gain cross section.

Finally, we investigated the waveguiding properties of the MC/PEMMA films upon optical gain. The guiding losses throughout the slab were determined by fixing the excitation length $(l=4.5 \mathrm{~mm})$ and by moving the pump stripe away from the emission edge of the sample, thus increasing the length $(d)$ of the unpumped region..$^{32,33}$ We found that the ASE output intensity decreases roughly exponentially upon

(31) Anni, M.; Gigli, G.; Cingolani, R.; Zavelani-Rossi, M.; Gadermaier, C.; Lanzani, G.; Barbarella, G.; Favaretto, L. Appl. Phys. Lett. 2001, $78,2679$.

(32) McGehee, M. D.; Gupta, R.; Veenstra, S.; Miller, E. K.; Diaz-Garcia, M. A.; Heeger, A. J. Phys. Rev. B 1998, 58, 7035.

(33) Heliotis, G.; Bradley, D. D. C.; Turnbull, G. A.; Samuel, I. D. W. Appl. Phys. Lett. 2002, 81, 415.

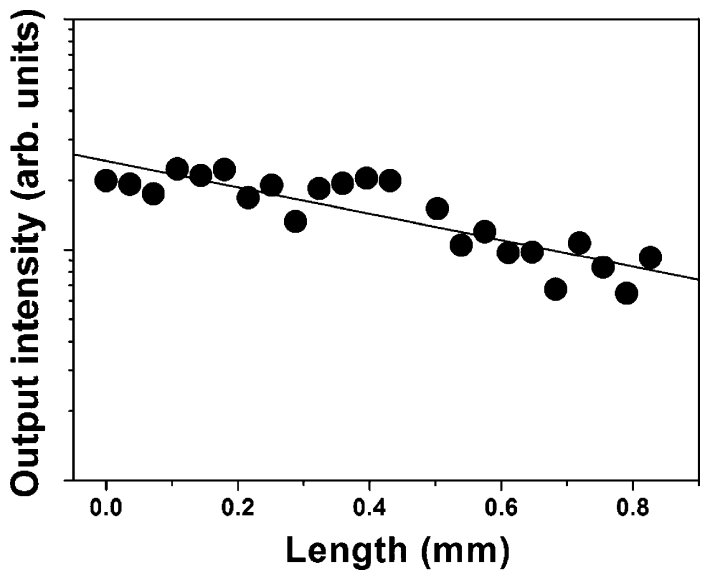

Figure 6. Dependence of the ASE output from the slab edge on the length (d) of the nonexcited region between the pump stripe and the edge of the sample. The dotted line is the best fit of the data to the equation $I=I_{0}$ $\exp (-\alpha d)$.

increasing $d$, thus suggesting the predominance of absorption losses (Figure 6). The measured loss coefficient of the MC waveguide is $\alpha=5.7 \pm 0.7 \mathrm{~cm}^{-1}$. This value is quite interesting, 1 order of magnitude lower than that found in the poly[2-methoxy-5-(2'-ethylhexyloxy)-1,4-phenylene vinylene], ${ }^{32}$ and of the same order of the best results reported for conjugated polyfluorenes ${ }^{33}$ and poly [ $(9,9-$ hexylfluorenyl-2,7ditl)-alt-co-(9-ethyl-3,6-carbazole)]s. ${ }^{34}$ The Stokes' shift for the investigated $\mathrm{MC}$ is indeed in the range $270-430 \mathrm{meV}$ (i.e., a wavelength distance between the absorption and the emission maxima, $\Delta \lambda$, of $80-140 \mathrm{~nm}$ depending on the emission spectral profile), determining low self-absorption losses.

The effectiveness of MC slabs as potential lasing systems is also confirmed by the fine lasing structures that we could appreciate in the ASE spectra under intense optical excitation. These peaks (fwhm of about $1 \mathrm{~nm}$ ) are, for example, observable in the spectra displayed in Figure 4, and their occurrence is usually attributed to feedback induced by randomly distributed scattering centers into inhomogeneous gain media. ${ }^{35}$

\section{Conclusion}

The occurrence of optical gain under long-pulse (nanosecond) pump conditions suggests that MC/PEMMA slabs are very promising active media for organic lasers operating under steady-state excitation and under high injection densities. Besides obtaining light amplification, reducing optical losses within the active media is also crucial for fabricating lasers. The here reported results (threshold excitation density for ASE observation $400 \mu \mathrm{J} \mathrm{cm}{ }^{-2}$, threshold pumping length in the range $0.5-1.2 \mathrm{~mm}$, and waveguiding losses as low as $5.7 \mathrm{~cm}^{-1}$ ) provide good indications that the photochromic system BIPS $\rightleftarrows$ MC can be employed for optical amplification and lasing. A possible $S_{1}$-dominated photoconversion and excitation/de-excitation dynamics is indeed compatible with the realization of efficient, possibly gateable, optoelectronic devices.

\section{CM060438J}

(34) Pisignano, D.; Mele, E.; Persano, L.; Paladini, G.; Cingolani, R. Appl. Phys. Lett. 2005, 86, 261104.

(35) Frolov, S. V.; Vardeny, Z. V.; Yoshino, K.; Zakhidov, A.; Baughman, R. H. Appl. Phys. Lett. 1999, 59, R5284. 